\title{
AEROSHELL DESIGN TECHNIQUES FOR AEROCAPTURE ENTRY VEHICLES
}

\author{
R. Eric Dyke \\ Swales Aerospace \\ NASA Langley Research Center, Hampton VA, USA \\ r.e.dyke@larc.nasa.gov \\ Glenn A. Hrinda \\ NASA Langley Research Center, Hampton VA, USA \\ g.a.hrinda@larc.nasa.gov
}

\begin{abstract}
A major goal of NASA's In-Space Propulsion Program is to shorten trip times for scientific planetary missions. To meet this challenge arrival speeds will increase, requiring significant braking for orbit insertion, and thus increased deceleration propellant mass that may exceed launch lift capabilities. A technology called aerocapture has been developed to expand the mission potential of exploratory probes destined for planets with suitable atmospheres. Aerocapture inserts a probe into planetary orbit via a single pass through the atmosphere using the probe's aeroshell drag to reduce velocity. The benefit of an aerocapture maneuver is a large reduction in propellant mass that may result in smaller, less costly missions and reduced mission cruise times. The methodology used to design rigid aerocapture aeroshells will be presented with an emphasis on a new systems tool under development. Current methods for fast, efficient evaluations of structural systems for exploratory vehicles to planets and moons within our solar system have been under development within NASA having limited success. Many systems tools that have been attempted applied structural mass estimation techniques based on historical data and curve fitting techniques that are difficult and cumbersome to apply to new vehicle concepts and missions. The resulting vehicle aeroshell mass may be incorrectly estimated or have high margins included to account for uncertainty. This new tool will reduce the guesswork previously found in conceptual aeroshell mass estimations.
\end{abstract}

\section{NOMENCLATURE}

B/S backshell

F/B forebody

FEA finite element analysis

FEM finite element model

GUI graphical user interface

JPL Jet Propulsion Laboratory

L/D lift to drag ratio

LV launch vehicle

MEM's mass estimation methods

NSM non-structural mass

TPS thermal protection system

VB visual basic

\section{INTRODUCTION}

Aerocapture vehicles belong to a group of Aeroassit technologies that use aerodynamic forces for maneuvering. During aerocapture, a vehicle

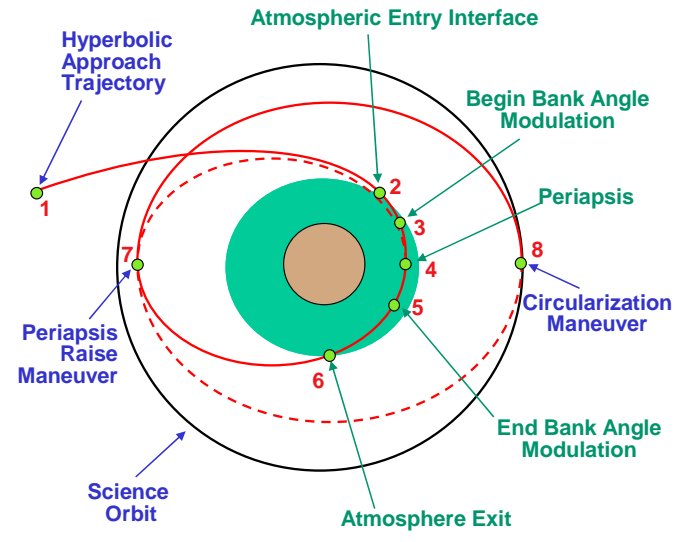

Figure 1. Aerocapture Overview

makes a single pass through an atmosphere, using the lift and drag characteristics of the vehicle's aeroshell (payload encasing structure) for deceleration and orbit insertion, as shown in Figure $1^{1}$.

This technology has shown potential advantages over chemical propulsion methods by reducing total vehicle mass, travel time, and mission $\operatorname{cost}^{1,2}$. 
Minimum propellant mass is required for orbit cleanup after aerocapture, resulting in either lower total launch mass or increased payload mass fractions for constant launch mass. Reducing total launch mass can reduce required launch vehicle (LV) size, thereby reducing launch costs.

Rigid aerocapture aeroshells can take a variety of general shapes, depending on the aerodynamic characteristics needed. Three typical shapes used in aerocapture case studies are the sphere-cone, biconic, and ellipsled, as shown in Figure 2. The sphere cone is considered a low $\mathrm{L} / \mathrm{D}$ shape, while the ellipsled and biconic are considered mid L/D shapes. The specific shape used is a function of aerodynamic and internal packaging volume requirements necessary for the given mission trajectory and destination atmosphere.

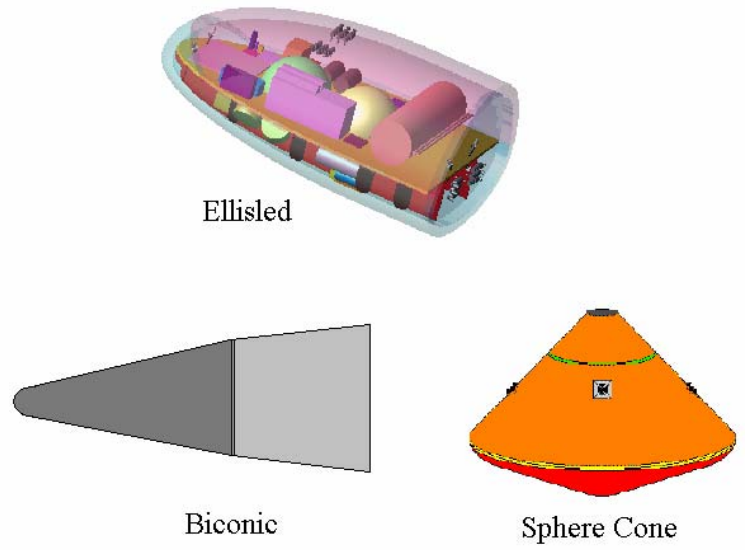

Figure 2. Aerocapture Aeroshell Shapes

Preliminary spacecraft systems studies have been performed for aerocapture missions to $\operatorname{Titan}^{1}$, Saturn's largest moon, and to Neptune ${ }^{2}$. These studies were done to identify and develop promising technologies for aerocapture systems analysis and help advance the state-of-the-art in mission planning and design. Both studies used rigid aeroshells for aerocapture, and delivered a science payload into orbit and lander/entry probe(s) into the atmosphere. Titan's aerocapture entry corridor allowed the use of a low L/D sphere-cone aeroshell ${ }^{3}$, while Neptune's entry corridor required a mid L/D ellipsled aeroshell ${ }^{4}$.

\section{AEROSHELL DESIGN METHODOLOGY}

A major part of these preliminary aerocapture system studies, as well as future conceptual aerocapture and direct entry studies, is providing quick and accurate aeroshell structure mass estimates. For both the Titan and Neptune studies, the same basic methodology was used in estimating rigid aeroshell structure mass. This basic methodology, as shown in Figure 3, would also be used for direct entry probes or any other mission variation requiring an aeroshell for atmospheric entry.

Trajectory analysis is used to define the required LV, associated payload capacity, travel time, and

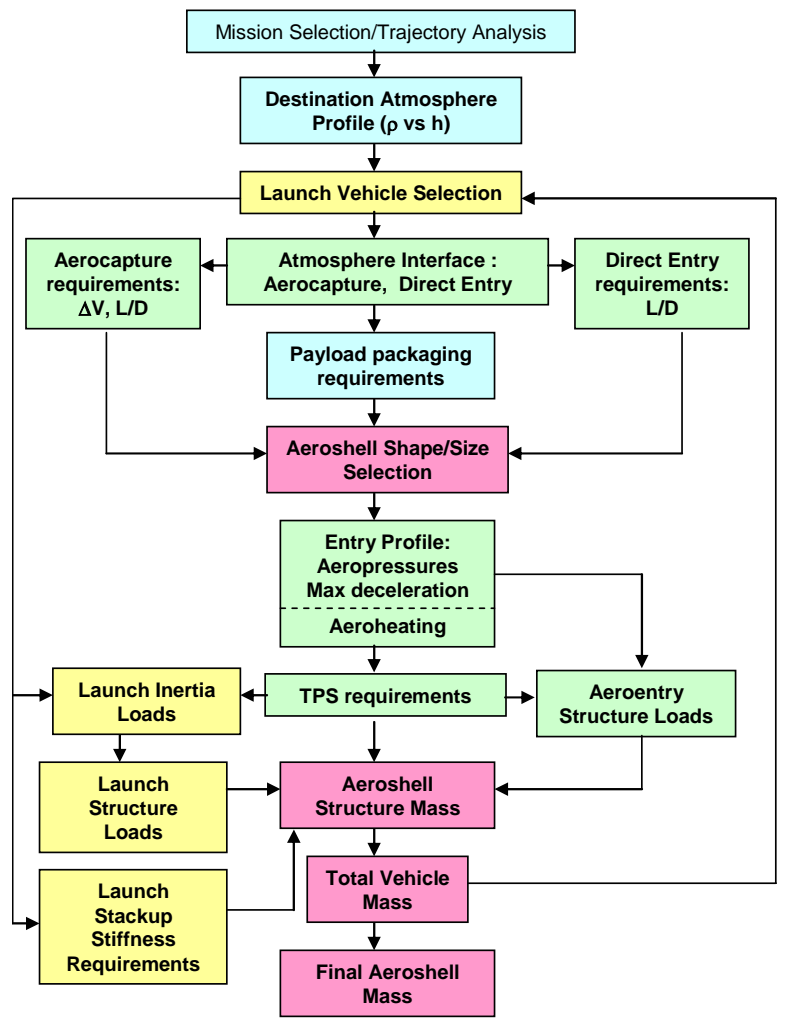

Figure 3. Aeroshell Design Flow

arrival velocity. The destination atmosphere model and entry profile help determine the general aeroshell shape required, while the payload requirements help determine overall aeroshell size. The LV sets requirements for launch stackup stiffness (natural frequency) and launch inertia loads. Aeroentry aerothermal analysis is used to define thermal protection system (TPS) material and thickness and to define applied aeroentry pressures and decelerations. Non-structural TPS mass, launch loads, and aeroentry loads are all used in conjunction with standard structural finite element analysis (FEA) to determine aeroshell materials, sizes, and mass. Throughout the process, iterations are made for payload mass and packaging changes, LV capability, trajectory and entry modifications, and applied loads. The result is a conceptual aeroshell design and associated mass. Further refinements to the design and structural analysis will result in a preliminary aeroshell mass. 


\section{SYSTEMS TOOL OVERVIEW}

Current methods for probe mass estimation, particularly aeroshell structure sizing and mass, often involve using separate, non-integrated historical data, mass estimation methods (MEM's), and finite element models ( FEM's) and structural sizing tools. Often the different aspects of conceptual probe sizing are done by different individuals using different platforms which may or may not share data easily ${ }^{5}$. The ultimate goal of any probe aeroshell sizing system tool would be to integrate mission and payload requirements, geometry engines, historical databases, MEM's, and structural FEA solutions into a seamless, end-to-end, user-friendly tool which can produce aeroshell (and total probe) mass estimates in a relatively short time.

In order to reduce conceptual design structural sizing time, and to increase the accuracy of estimated aeroshell structure mass, a systems tool called ProbeMASS1 is currently being developed. This tool integrates three commercially available software packages (Microsoft ${ }^{\circledR}$ Excel $^{6}$, EDS PLM Solutions I$\mathrm{DEAS}^{7}$, and Collier Research's HyperSizer ${ }^{\mathrm{TM}^{8}}$ ), historical aeroshell data, unique Excel Visual Basic (VB) code macros, and MEM's into a user-friendly probe mass estimation tool which focuses on aeroshell structural sizing. The primary benefit of ProbeMASS1 over other methods, when complete, will be to allow a single user to quickly and accurately estimate conceptual aeroshell mass utilizing a single tool in a relatively short time ${ }^{5}$.

The schematic in Figure 4 shows ProbeMASS1's three major software platforms and their respective primary functions. ProbeMASS1 is currently limited to sphere-cone and ellipsled type aeroshells. Future versions will address other shapes such as biconics and bent biconics. While ProbeMASS1 will estimate various subsystem masses, the general payload packaging must be known/given to select a reasonable initial aeroshell size. Also, atmosphere entry parameters such as velocity, angle, aeroheating, etc., must be determined outside of this tool. Lastly, the automated structural sizing is limited to the basic aeroshell only. Internal structure, such as payload support trusses, etc. must be handled offline and the resulting mass added to the total mass.

A unique feature of the systems tool is a detailed, well-referenced historical database that lists a variety of system design information from previously flown missions or conceptual studies. This feature enables a systems engineer quick access to design information that may be incorporated into a new mission. The historical database will be explained in more detail in a later section.

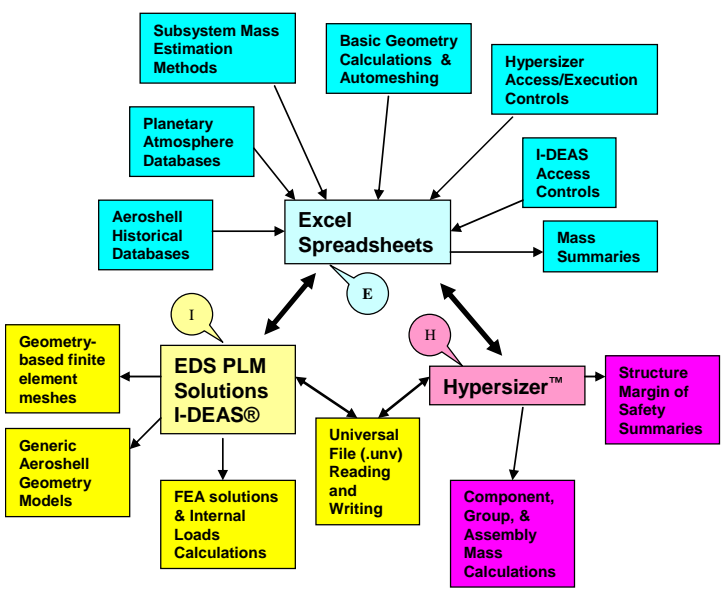

Figure 4. ProbeMASS1 Platform Schematic

The schematic in Figure 5 shows the work flow path when performing an end-to-end aeroshell sizing with ProbeMASS1. The solid boxes represent interactive work done in the Excel platform. The long dashed boxes represent interactive work done in the I-DEAS platform. The short dashed boxes represent non-interactive (batch) operations within the HyperSizer ${ }^{\mathrm{TM}}$ platform.

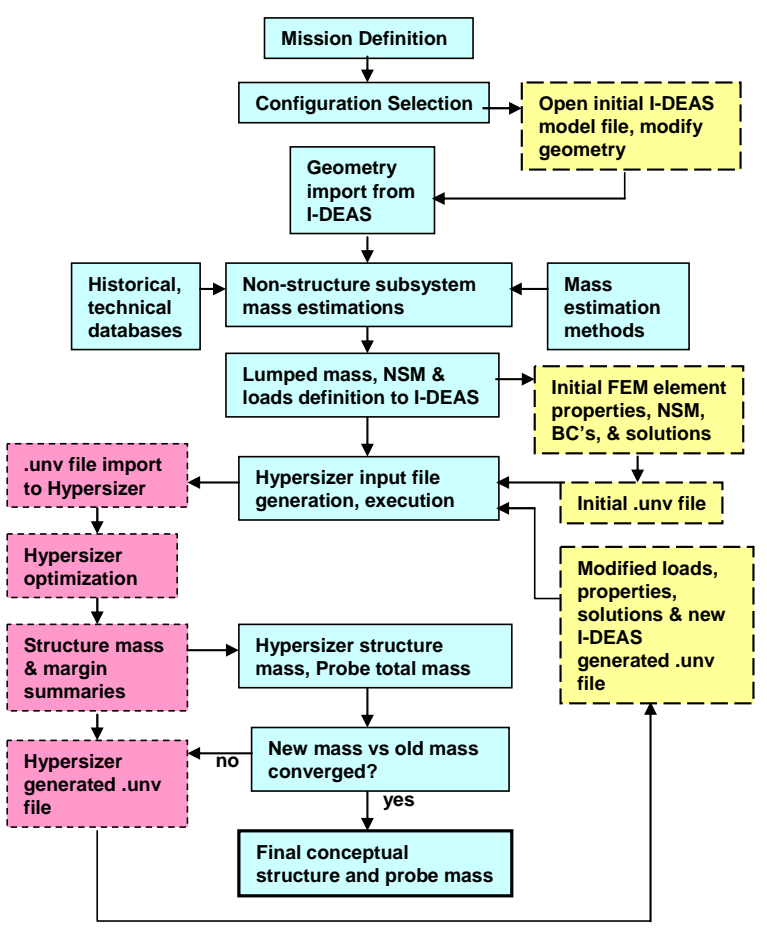

Figure 5. ProbeMASS1 Functional Schematic

\section{Excel Platform}

The main platform for the systems tool is an Excel Workbook called ProbeMASS1. This Workbook contains worksheets, as shown in Figure 6, which 
estimate various subsystem masses for sphere-cone and ellipsled vehicles, contain historical and other databases, and provide automated links to transfer data to and from the other two platforms. The circled letters represent data transfer to/from the I-DEAS (I), Excel (E), or HyperSizer ${ }^{\mathrm{TM}}(\mathrm{H})$ platforms.

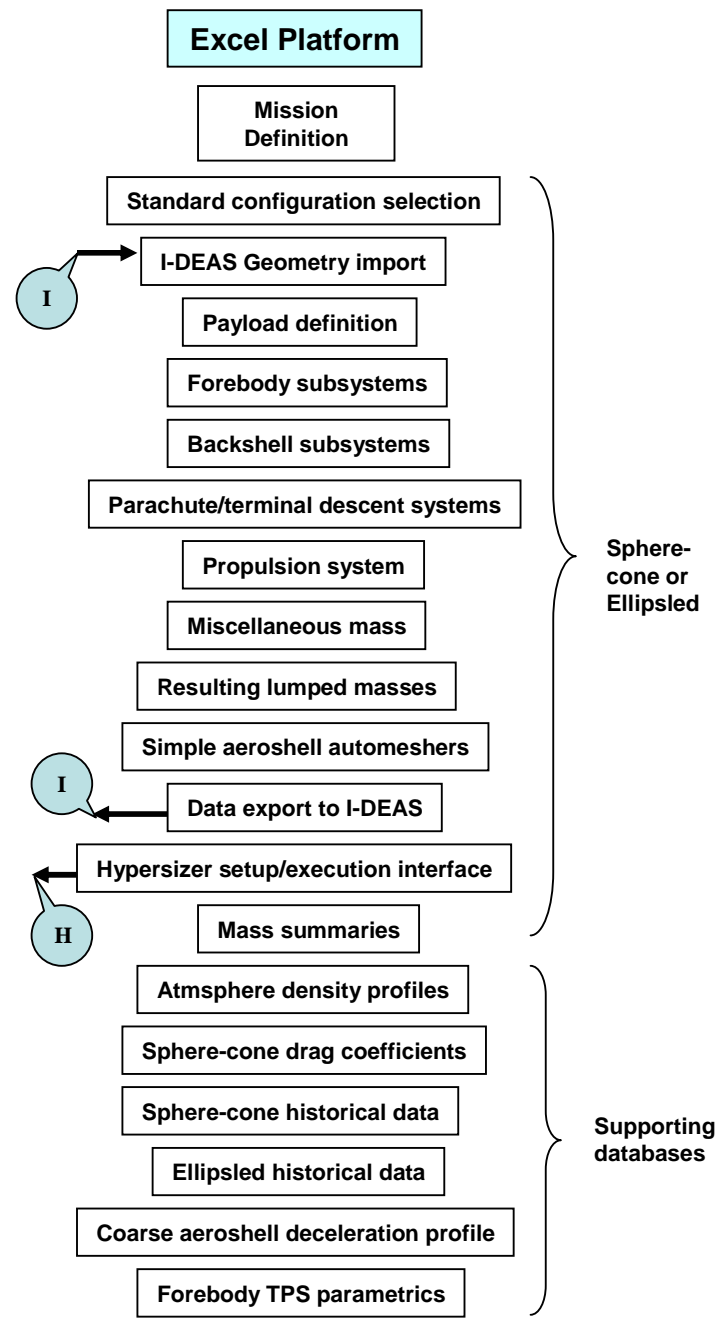

Figure 6. Excel Platform Schematic

\section{$\underline{\text { Standard Geometries }}$}

The user must start with a given or assumed mission profile, including destination, science objectives, and atmospheric entry profile. The full entry profile is determined from external mission or entry analysis. The required payload packaging to meet the science objectives, combined with the entry velocity, entry angle, and required L/D to maintain the entry corridor, determine the overall required aeroshell shape and initial aeroshell size. The user selects from a set of standard geometries (spherecone or ellipsled only at this time), then executes an Excel VB macro to open a new I-DEAS model file and import the standard geometry. Generic functions in I-DEAS are automated through the use of I-DEAS program files which are written, modified and executed by the Excel VB macros, or executed by the user. The user interactively modifies the basic geometry to the specific geometry needed for the current mission. Automated on-screen text prompts guide the user through the various processes in IDEAS. The file is saved, and the aeroshell dimensions and surface area data are imported into the appropriate Excel worksheets, again through the use of I-DEAS program files and Excel VB macros.

\section{Subsystem Mass Estimation}

After geometry import, the user steps through a series of interactive Excel worksheets, as shown in Figure 6, to estimate various system and subsystem masses. Within each of these major systems are associated subsystems. For example, the Forebody (F/B) System includes aeroshell structure (low fidelity), TPS, separation, thermal control, harness, and miscellaneous subsystem mass estimation. For each subsystem, various MEM's are used. For the F/B and backshell (B/S) structure, a coarse, first-pass mass estimate can be made based on trend curves from previous probe designs, or the more accurate finite-element-based estimation method can be used. The other mass estimates take several forms, depending on the particular system or subsystem, including using values from historical data, simple parametric scaling (area or length scaling), or high fidelity, standard closed-form solutions, particularly for propulsion subsystems.

To make the worksheets more user-friendly, a consistent color coding system is used throughout the Excel platform. By simply scanning any given worksheet, the user can tell at a glance the type of data contained in a cell or if user interaction (yellow cells) is required. When completed, the tool will lock all cells but those requiring user input.

Finite Element Solution

If a low fidelity, first pass structure and probe mass are desired, the user may simply step through the subsystem MEM's listed above, and the resulting masses will be detailed in a probe mass summary sheet. For a more accurate mass estimate, particularly for the aeroshell structure mass, the FEA based solution must be used. Once the user has stepped through all the required subsystems for the given mission, the resulting non-structure component masses are lumped into F/B-associated (payload, etc.) and B/S-associated (parachute, etc.) masses. Harness, thermal control, and TPS masses are converted to non-structural masses (NSM's) and assigned to their respective location on either the $\mathrm{F} / \mathrm{B}$ 
or B/S. The user then defines the applied launch inertia loads and aeroentry inertia loads. Several zones of constant pressure are used to balance the aeroentry inertia and to provide for a simple pressure distribution across the aeroshell forebody surface. The user can modify the distribution of pressure to help match pressure peaks as determined from separate aerothermal analyses. The applied loads, lumped masses and NSM's are then automatically read into the previously created I-DEAS model through the use of I-DEAS program files and Excel VB macros. Within I-DEAS automatically generated on-screen text prompts guide the user to attach the lumped masses to the aeroshell, create appropriate FEM boundary conditions, run static solutions to generate internal FEM loads, and create an I-DEAS universal file for import into HyperSizer ${ }^{\mathrm{TM}}$. The IDEAS and HyperSizer ${ }^{\mathrm{TM}}$ platforms and their functions will be detailed more in their respective sections.

\section{HyperSizer ${ }^{\mathrm{TM}}$ Interface}

After the FEA is completed in I-DEAS, control is returned to the Excel worksheet where the user then sets up an input file for HyperSizer ${ }^{\mathrm{TM}}$. The input file provides a means of setting up all required file management, solution sequences, load set definition, Group \& Assembly generation, and NSM control for a batch run of HyperSizer ${ }^{\mathrm{TM}}$. An Excel VB macro controls the HyperSizer ${ }^{\mathrm{TM}}$ execution and creates separate text files with debug, mass, margin of safety, and NSM definition summaries. This macro is adapted for use in Excel VB from an existing VB6 HyperSizer $^{\mathrm{TM}}$ interface code called HSLoad ${ }^{9}$. The macro also returns real-time status of the HyperSizer ${ }^{\mathrm{TM}}$ run and the final aeroshell structure mass to the Excel worksheet for use in the full probe mass summary, shown in Figure 6 . On the HyperSizer $^{\mathrm{TM}}$ interface worksheet, masses from previous and current structure iterations are tracked and used to determine solution convergence when iterating between I-DEAS and HyperSizer ${ }^{\mathrm{TM}}$. The mass summaries worksheet lists all system and subsystem masses for the full probe, including the aeroshell structure mass.

\section{Database Worksheets}

In addition to the mass estimation worksheets, the Excel file also contains several database sheets, as shown in Figure 6. The Celestial Body Database sheet contains simple atmospheric density vs. altitude profiles for all the known atmosphere-bearing planets and moons in the solar system. Some bodies have several detailed profiles, based on past earth-based and in situ observations, while others have only one coarse profile based on best available data. These profiles can be used in conjunction with the drag coefficient database sheet and the aeroshell deceleration sheet to produce stand-alone maximum entry deceleration estimates. These stand-alone estimates can be helpful for quick mass estimation tasks when external entry profile analyses are not readily available.

The sphere-cone and ellipsled database sheets contain extensive historical data on previous flight and system study planetary probes. They contain mostly data from NASA-directed probes, but also a limited amount of data for European, Soviet, and Japanese probes. The data are grouped according to probe destination, and are further subdivided into the major system and subsystem categories shown in Figure $7^{5}$. The first four categories are probe overviews: Mission Overview (destination, launch

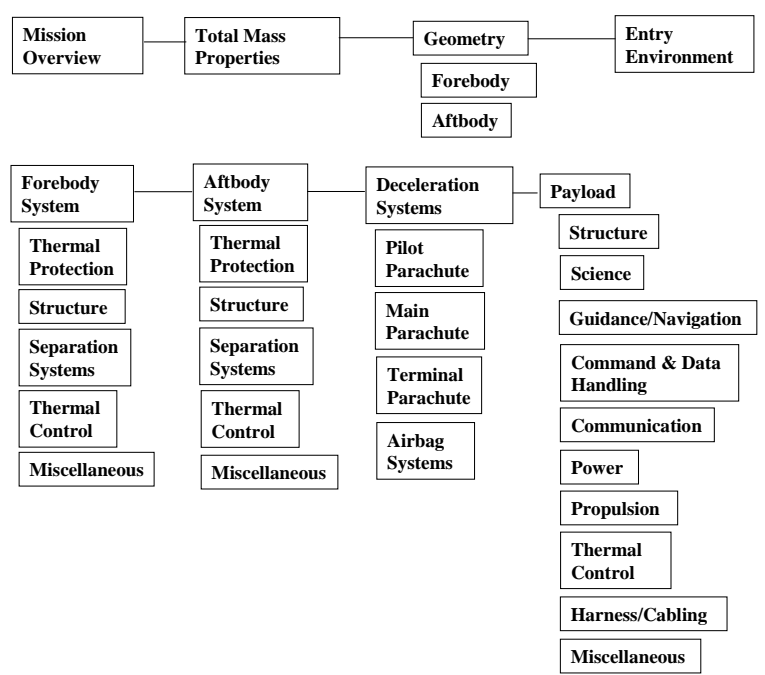

Figure 7. Historical Database Breakdown

date, LV), Total Mass Properties (mass, moments of inertia), Geometry (overall F/B and B/S dimensions), and Entry Environment (speed, angle, maximum deceleration, maximum heat rate and heat load). Following these are the major probe systems of $\mathrm{F} / \mathrm{B}$, B/S (or aftbody), deceleration system (parachutes), and payload, and their associated subsystems. The same general breakdown is followed for the spherecone and ellipsled vehicles, though considerably less ellipsled data is available.

Every cell or every line of cells in the historical databases contains a cell comment that lists a reference for the data shown. For cases where no data was found, the cell was left blank. In other cases, conflicting values were found in the literature. In such cases the most recent value was entered in the cell while the older data and reference were still listed in the cell comment. In addition, the cell comments are used to include more detailed data, such as sandwich panel dimensions, that can not 
easily be listed in the cells. Lastly, some cell data is calculated from other found data (ballistic coefficient, for instance, based on mass, diameter, and drag coefficient), and uses tan color coding to indicate a calculated value.

The final database sheet is the Forebody TPS Parametrics sheet. TPS sizing involves very complex analysis, and does not lend itself easily to scaling. The TPS database contains detailed information on previous point TPS analyses for various flight probes and paper studies. The resulting sizings from these analyses were used to develop very coarse and limited TPS MEM's for future conceptual probe designs. Different parametrics were used for mass estimation for a given atmosphere, such as heat load, entry angle, or ballistic coefficient, based on the limited data available. These scaling laws are very primitive and overly simplistic and lose reliability for new designs which differ greatly from the previous designs on which the original TPS mass was based. While they can be used for conceptual design, caution should be used when trying to extrapolate too far beyond the original designs.

\section{Aeroshell Automeshers}

Early in the tool development, automeshing routines were added to the Excel spreadsheets for the sphere-cone and ellipsled shapes. These routines created basic aeroshell finite element meshes using plate elements only. Separate F/B and B/S NSM's were calculated from TPS data and added to the plate element properties. Simple axial inertia and uniform zonal aeropressure loading could also be specified by the user as part of the automeshing routine. Excel VB macros then created NASTRAN ${ }^{10}$.dat files of the mesh data which could easily be imported into a variety of structural FEM pre- and post-processors, such as FEMAP, I-DEAS, or PATRAN. Once in the pre-processor, the user was required to add the unique boundary conditions (grounding restraints and F/B-B/S constraints), lumped masses, and rigid elements joining lumped masses to the aeroshell. Due to the relatively simplistic nature of the spherecone geometry, nearly any sphere-cone forebody and backshell configuration could be modeled, with up to six conic "zones" and a (hemi)sphere cap on the backshell. Figure 8 shows a sphere-cone mesh with a biconic backshell, and a simple ellipsled mesh ${ }^{5}$. For the more complex ellipsled geometries, sixteen different basic configurations were allowed to account for different types of backshell separation geometries.

And while the sphere-cone geometry allows closed-form methods for calculating surface areas and volumes, ellipsoid geometry does not. The ellipsled automeshing VB macros allow the user to create a basic ellipsled shape, then calculate surface areas and volumes from the discrete elements used in the mesh.
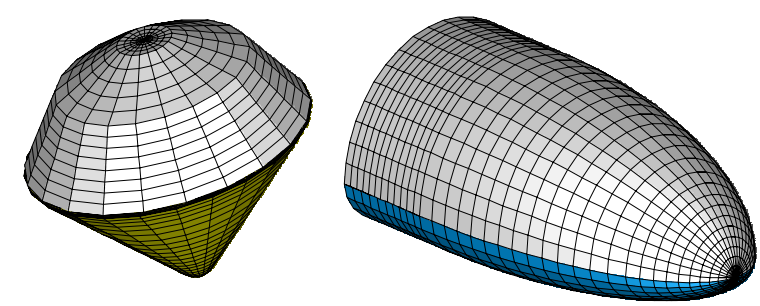

Figure 8. Automeshed Sphere-Cone and Ellipsled Aeroshells

This feature allows for rapid mass calculations for any item based on surface area, such as TPS and, in very coarse instances, structure.

Later in the tool development, standard I-DEAS geometry models and meshes were integrated with the EXCEL features, thereby reducing the need for the EXCEL-based automeshing or surface area calculations. However, the automeshing feature was retained because it allows quick, stand-alone meshing and surface area/volume calculations which can be used to estimate piecemeal masses, as described in more detail in the Applications section of this paper.

\section{$\underline{\text { I-DEAS Platform }}$}

EDS PLM Solutions I-DEAS (Integrated Design, Engineering, and Analysis Software) is a

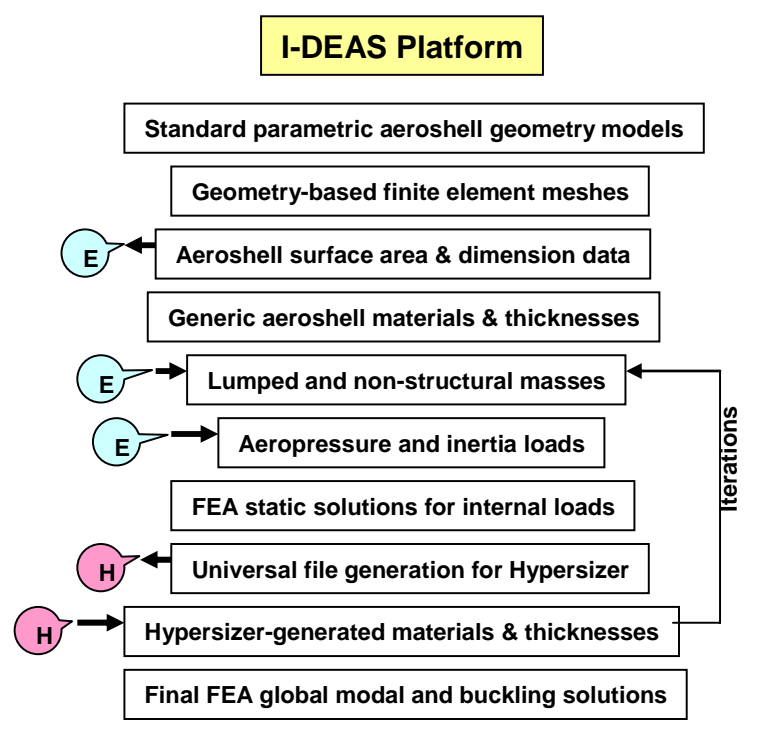

Figure 9. I-DEAS Platform Schematic

commercially available software package. It is a collection of applications (design, simulation, and manufacturing) that share a common master solid model $^{11}$. The I-DEAS platform, as shown in Figure 
9, is used to maintain standard aeroshell geometry and FEM mesh files, and to solve structural static, modal, and buckling solutions and provide element internal static loads within the ProbeMASS1 tool.

\section{Standard Geometries}

Several standard aeroshell geometry models have been/will be created for sphere-cone and ellipsled type vehicles. For the sphere-cone, the standard geometries contain the basic sphere-cone forebody and different backshell configurations and forebody/backshell interface planes. Conic, biconic, and triconic, and (hemi)sphere-cap backshells are standard. These backshells can be joined to the forebody either at the shoulder radius tangency, or farther aft, creating a forebody shoulder cone. Payload rings or payload decks are also standard payload/forebody interface structure. New standard configurations can be added to the database as required.

The standard geometry models are held in an IDEAS library. When the user activates the Excel VB macro to open a standard model, it automatically retrieves the model from the library, saves it as a new model file with the user-specified name, then cuts association with the library so that the standard models can not be altered by users.

For the ellipsled, currently one basic aeroshell geometry is available. Due to the more complex nature of the ellipsled aeroshell and internal structure configurations, only the simplest conceptual aeroshell configurations can be automeshed at this time. More standard ellipsled configurations will be added as relevant designs become available.

\section{Geometry-based Meshes}

Sphere-cone and ellipsled structural finite element meshes have been/will be created and associated with their respective geometries. One feature of I-DEAS allows geometry-based meshes to be modified automatically when the geometry is modified. Therefore, when a user opens a standard geometry file and updates the dimensions for a specific vehicle and mission, the mesh will automatically update as well. These default meshes have "dummy" materials and element properties (thicknesses) for use in the initial I-DEAS finite element analysis. Results from the initial default solution are fed into HyperSizer ${ }^{\mathrm{TM}}$ as described in a later section.

Once the standard geometries are modified to a specific vehicle by the user, the associated geometry (dimensions and surface areas) is imported automatically into the Excel spreadsheets using IDEAS program files and Excel VB macros. This geometry data is used throughout the Excel worksheets as part of the subsystem MEM's discussed earlier.

After the other subsystem masses have been estimated in the Excel spreadsheets, the lumped masses and aeroshell NSM's are automatically transferred back to I-DEAS, again using Excel VB macros and I-DEAS program files. In addition to the masses, aeropressure and inertia loads (launch and aerocapture g loads) are transferred. At this point, the user is required to create the unique boundary conditions, tie the aeroshell forebody and backshell together, tie the lumped masses to the aeroshell, and run the required static solutions for the different load conditions previously defined. Throughout this process, on-screen text prompts guide the user through the various steps and in the selection of various I-DEAS program files to help automate the process. After running all solutions, the user is prompted to execute a program file which creates an I-DEAS universal file which will be imported into HyperSizer $^{\mathrm{TM}}$. After the universal file is created, control is returned to the Excel platform where preparations are made to run HyperSizer ${ }^{\mathrm{TM}}$.

\section{HyperSizer $^{\mathrm{TM}}$ Platform}

\section{What is HyperSizer ${ }^{\mathrm{TM}}$ ?}

HyperSizer $^{\mathrm{TM}}$ is a non-deterministic structural sizing software package developed by Collier Research Corporation $^{8}$. It allows the user to evaluate numerous detailed structural concepts in a short time with a single coarse-grid FEM. This approach greatly reduces the time otherwise necessary to mesh structural details, like stiffeners, required for other standard FEA solutions. The user must judiciously select element materials and properties for different regions of the model, called "panels". The "panel”, or "Component" is the basic analysis block in HyperSizer $^{\mathrm{TM}}$. HyperSizer ${ }^{\mathrm{TM}}$ automatically creates these Components by lumping all elements with a given material/property combination into a panel, then using the internal element loads from an outside solution (I-DEAS for this tool) to perform closedform and statistical strength, stiffness, and stability checks on that panel. These Components are then put into Groups which would share common construction methods or common design variables. A given Component can only be part of one Group. In addition, Assemblies can be created using any combination of Components and Groups in order to track mass for larger portions of a structure, or for the entire structure. While not a true optimizer, HyperSizer $^{\mathrm{TM}}$ steps through a user-defined design space of possible structural concepts (sandwich, blade stiffened, isogrid, etc., panels) and structural details (sheet thickness, blade height, stiffener 
spacing, etc.) to determine the lightest permutation which satisfies all the strength and stiffness criteria.

\section{Execution Formats}

HyperSizerTM can be run in two ways. The first is the standard graphical user interface $(\mathrm{GUI})^{8}$, which is a series of windows/tabs that include file management and load set manipulation, and that allow the user to set up the design space for each Component/Group interactively. The second is a batch method using Object Model Programming ${ }^{12}$. In this method, all file and load set management and design space definition is done through the use of input files and external execution code (Excel VB in this tool). ProbeMASS1 primarily uses the batch method, but the user has the ability to review the setup or solution at any time using the GUI method, and has the ability to use an initial model from ProbeMASS1 for more extensive offline analyses.

\section{Integration with ProbeMASS1}

The HyperSizer ${ }^{\mathrm{TM}}$ functions within ProbeMASS1 are shown in Figure 10. The HyperSizer ${ }^{\mathrm{TM}}$ solution starts with the setup/execution input file creation in Excel. The user specifies all relevant files and file paths for file management. In addition, the user specifies the load cases to be used from the I-DEAS solutions, creates groups and assemblies as desired/required, and lists the relevant Global Group Design to be used for each group. Global Group

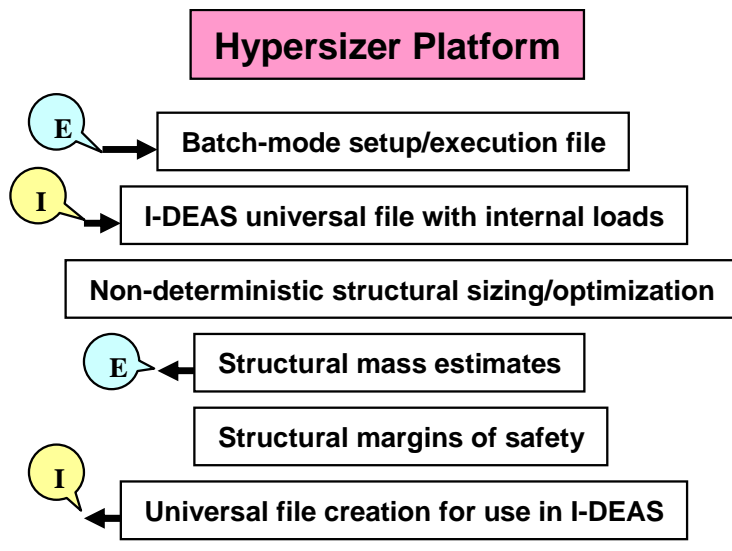

Figure 10. HyperSizer ${ }^{\mathrm{TM}}$ Platform Schematic

Designs are pre-defined design spaces set up for each group. They contain the basic construction method (Family), such as sandwich, stiffened panel, isogrid, etc. They also contain the ranges of design variables used in the design space, such as the different materials to be considered, the minimum and maximum face sheet thicknesses, and number of thickness steps for sandwich facesheets. Default Global Group Designs for sandwich construction exist for sphere-cone and ellipsled analysis. The user may also enter new Global Group Designs prior to running the HyperSizer ${ }^{\mathrm{TM}}$ portion of ProbeMASS1 by using the HyperSizer ${ }^{\mathrm{TM}}$ GUI and setting up desired design spaces.

An Excel VB macro automatically creates a suitable HyperSizer ${ }^{\mathrm{TM}}$ execution input file from the user-specified data. This input file is read by a second Excel VB macro which opens HyperSizer ${ }^{\mathrm{TM}}$ in batch mode, reads in the mesh and internal loads from the I-DEAS generated universal file, creates the Groups and Assemblies, applies the Global Group Designs, defines element NSM's, then steps through all the design space permutations and finds the lightest structure, on a Component-by-Component basis, to meet all design criteria.

After sizing is completed, HyperSizerTM lists all component minimum margins of safety and all Components with negative margins of safety. The Excel VB macros also sort through the HyperSizer ${ }^{\mathrm{TM}}$ results and determine "over designed" Components by searching for those with positive margins of safety and sized to the first design space permutation. Such Components are using the minimum gages specified and can theoretically be made lighter by changing the design space (Global Group Design). The resulting structure mass and any over/under designed components are listed in the HyperSizer ${ }^{\mathrm{TM}}$ interface spreadsheet in Excel, as well as separate automatically generated output text files.

The HyperSizer ${ }^{\mathrm{TM}}$ structure mass must be compared to the initial structure mass with the default materials and properties from the standard aeroshell models. While the new mass is sufficiently different from the old, or while the internal loads are changing significantly due to large changes in component structure stiffnesses, the structure must be iterated on to arrive at a converged solution. To facilitate this, HyperSizer $^{\mathrm{TM}}$ automatically creates an I-DEAS universal file with updated element material and stiffness matrix data. This universal file can then be imported into I-DEAS and the applied aeropressures updated (if necessary) automatically using the Excel VB macros and I-DEAS program files. The static solutions are then rerun, and a new I-DEAS universal file created, again using an existing program file. This new universal file is imported back into HyperSizer $^{\mathrm{TM}}$, this time using a different Excel VB macro which preserves all the original Component, Group, Assembly, and NSM data, and which only reads in new internal loads. HyperSizer ${ }^{\mathrm{TM}}$ is executed and new masses are calculated. This process is repeated until the aeroshell structure has converged sufficiently, based on user-defined criteria. Throughout this iteration cycle, I-DEAS and HyperSizer $^{\mathrm{TM}}$ generated universal file names are automatically incremented and HyperSizer ${ }^{\mathrm{TM}}$ 
generated masses are automatically tracked within ProbeMASS1.

\section{HyperSizer $^{\mathrm{TM}}$ Limitations}

While HyperSizer ${ }^{\mathrm{TM}}$ will step through a large structure with a large design space relatively quickly, its optimization is panel specific. Adjacent panels may have completely different designs based on their loading and the HyperSizer ${ }^{\mathrm{TM}}$ sizing. This may give a minimum mass design for the entire structure, but it may not be a manufacturable one. At this point, the user may opt to accept this theoretical minimum mass design for use in broader, more conceptual trade studies, or they may wish to refine it to a more preliminary, realistic, manufacturable one. In the latter case, the user must either revise the original finite element mesh, "link" the components within HyperSizer ${ }^{\mathrm{TM}}$, or "force" the design to a single permutation for several components within HyperSizer $^{\mathrm{TM}}$. The first method requires modifying the original mesh, while the other two require either offline (GUI) runs of HyperSizerTM (for the "linking" procedure), or modifying the Global Group Designs which contain the design space.

HyperSizer $^{\mathrm{TM}}$ only performs stability and natural frequency checks on local panels. As a final check, the user should re-import the final HyperSizer ${ }^{\mathrm{TM}}$ generated universal file into I-DEAS and perform global buckling and natural frequency analyses to ensure the structure meets all design criteria. In some instances, the Component-by-Component sizing method produces a minimum mass structure that is not globally stiff enough to satisfy natural frequency or global buckling criteria. In such cases, the user generally must rely on I-DEAS solutions and engineering judgment to stiffen the structure adequately, then re-evaluate it in HyperSizer ${ }^{\mathrm{TM}}$ for detailed strength margins of safety using updated design space data.

\section{APPLICATIONS}

\section{Titan Aerocapture 2002}

While still in development, and intended for more conceptual design mass estimates, portions of ProbeMASS1 have been successfully used to support several systems studies requiring preliminary mass results. Figure 11 shows aeroshell TPS mass sensitivities calculated for a Titan sphere-cone aerocapture orbiter ${ }^{13}$ as part of a Titan Aerocapture Systems Analysis study in 2002. As part of that same study, aeroshell mass sensitivities relative to aeroshell diameter were calculated, as shown in Figure 12. The geometry calculators in ProbeMASS1 were used in conjunction with externally calculated TPS areal densities ${ }^{14}$ to estimate total TPS mass. Since the integrated aeroshell structural sizing was not incorporated at that time, an external FEM-based aeroshell structure sizing ${ }^{15}$ was used with simple scaling laws to estimate aeroshell structure mass vs. size. The numerous configurations were quickly run through ProbeMASS1 and the results used to produce the figures shown here.

Also as part of the Titan aerocapture study,

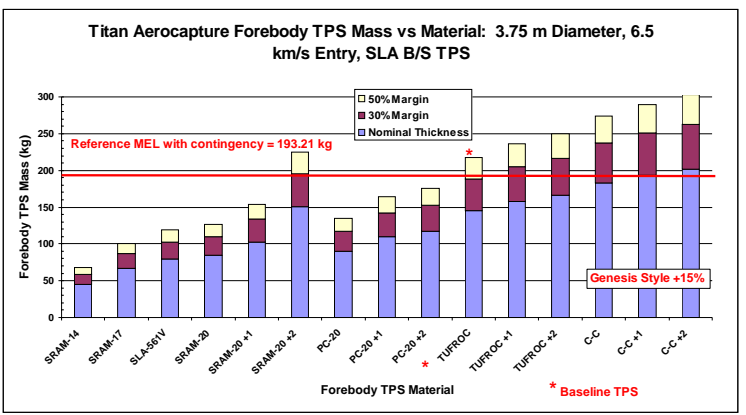

Figure 11. Titan Aerocapture TPS Mass Sensitivities

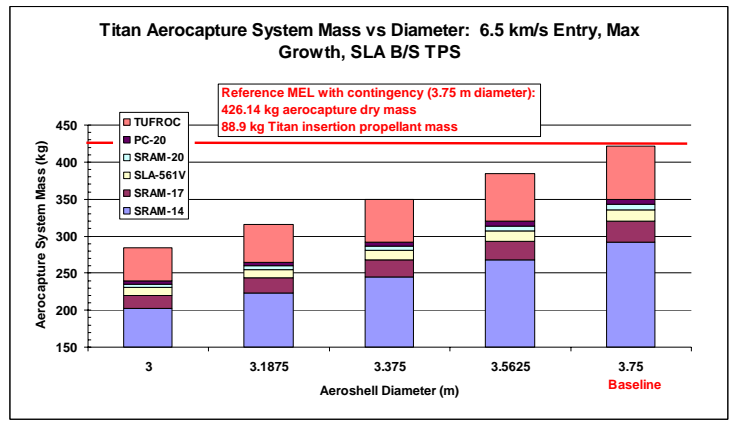

Figure 12. Titan Aerocapture Aeroshell Mass vs. Diameter

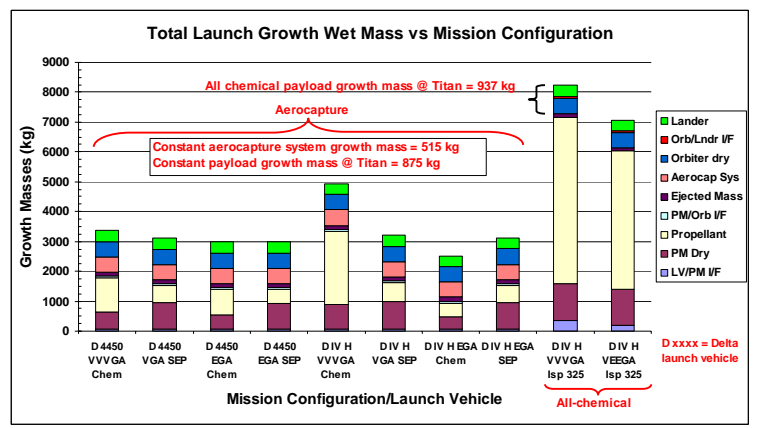

Figure 13. Titan Aerocapture Total Launch Mass vs. Mission Configuration

several trades were run to calculate total launch mass variations with launch vehicle and in-space propulsion system differences. The propulsion system worksheet in ProbeMASS1 was used to quickly estimate propulsion system masses for the various configurations. The resulting propulsion 
mass data, in conjunction with other externally calculated system masses ${ }^{16}$, were used to produce the sensitivity results shown in Figure 13.

\section{The Titan Project (JPL) 2003}

A second systems study using ProbeMASS1 worksheets estimated sphere-cone aeroshell masses for several Titan entry missions ${ }^{17}$. Four basic missions (orbiter aeroshell for aerocapture, lander aeroshell for direct entry, large aeroshell around lander and orbiter for aerocapture, and aeroshell around lander for entry from orbit) were evaluated for three different entry speeds (5.1, 5.9, and 6.5 $\mathrm{km} / \mathrm{s}$ ). To assist in the short study timeframe, payload packaging and entry profiles (maximum g loads, heating) were performed outside of ProbeMASS1. Historical TPS data from the 2002 Titan Aerocapture study discussed previously was scaled and used with the ProbeMASS1's geometry calculators to estimate TPS mass. The externally supplied packaging designs were used to determine overall aeroshell sizes. Entry profile data (g loads) were used with the simple aeroshell structure mass trend lines in ProbeMASS1 to estimate aeroshell structure masses. The analyses were run for various ballistic coefficients, and the results plotted as shown in Figure 14.

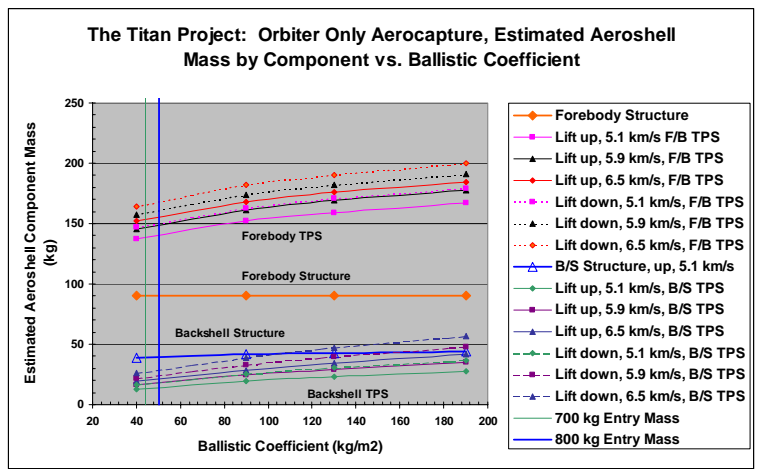

Figure 14. The Titan Project Aeroshell Mass Estimates

\section{Neptune Aerocapture 2003}

The most recent systems study using ProbeMASS1's capabilities was the Neptune Aerocapture Systems Study of 2003. Neptune aerocapture required a mid $\mathrm{L} / \mathrm{D}$ vehicle ${ }^{4}$, so a flattened ellipsled shape was selected ${ }^{18}$. The complex interactions between payload packaging, payload support, and aeroshell geometry and function quickly became apparent in this study ${ }^{19,20}$, requiring much more detailed finite element modeling than is currently available in ProbeMASS1. However, the ellipsled automesher and surface area calculator within ProbeMASS1 was extensively used to quickly calculate aeroshell surface areas for several aeroshell sizes and TPS configurations (varying zones of TPS thickness), helping define a final size required to meet launch mass budgets. These surface area results were also used in conjunction with externally calculated TPS thicknesses and areal densities ${ }^{21}$ to provide TPS mass sensitivities for the systems study. Figure 16 shows a sample of the system mass sensitivities $^{22}$, where the $\mathrm{x}$-axis labels represent various combinations of TPS materials and aerothermal heating.

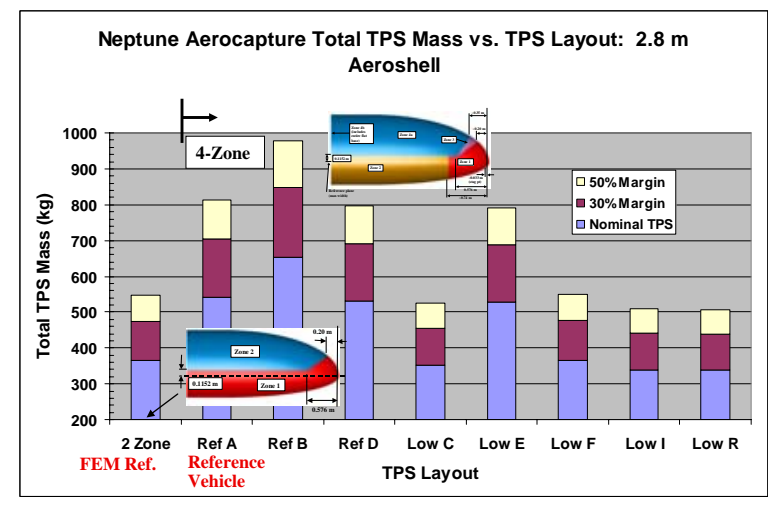

Figure 15. Neptune Aerocapture TPS Mass Sensitivities

\section{CONCLUSIONS}

The systems analysis tool, ProbeMASS1, is still in development. While it is intended as a conceptual design tool, parts of it have been used with great success to provide quick mass estimates for structure and TPS for various studies requiring higher fidelity preliminary results. It has also been successfully used to generate mass estimates for numerous iterations and configurations in a short time. When finished, this tool will provide the systems engineer a quick method for generating more accurate aeroshell structure and complete probe mass estimates. Uncertainty margins in a vehicle's mass estimation will be reduced and the mission's final results on $\mathrm{LV}$ lift requirements, costs and reliability will be enhanced. System designers will benefit from this tool by: being able to compare alternative mission technologies within an unbiased framework; having access to design information from previous missions; using a familiar spreadsheet format and a minimum number of separate software packages for a userfriendly environment allowing quick analysis; having links to other state-of-the art analysis programs for sizing composite structural members and estimating 
aeroshell mass. The tool will bring accurate aeroshell and total probe mass estimates to systems designers in the quickest time so decisions can be made with a higher level of fidelity and confidence.

\section{ACKNOWLEDGEMENTS}

The author wishes to thank Dr. Mary Kae Lockwood, K. Chauncey Wu, and Glenn Hrinda of the NASA Langley Research Center's Vehicle Analysis Branch for their guidance in developing the tool requirements and architecture. The author would also like to thank Shawn A. Krizan of Analytical Mechanical Associates for his assistance in I-DEAS solid modeling, and Dr. Lloyd B. Eldred of Swales Aerospace for his assistance with the HyperSizer ${ }^{\mathrm{TM}}$ Visual Basic interface.

\section{REFERENCES}

${ }^{1}$ Lockwood, M.K., et al, Overview, Neptune Aerocapture Systems Analysis Review, Marshall Space Flight Center, Huntsville, AL, October 28, 29, 2003.

${ }^{2}$ Lockwood, M.K., et al, Titan Aerocapture Systems Analysis Overview, Titan Aerocapture Systems Analysis Review, Jet Propulsion Laboratory, Pasadena, CA, August 29-30, 2002.

${ }^{3}$ Starr, B.R., Powell, R.W., Top Level Sensitivities, Titan Aerocapture Systems Analysis Review, Jet Propulsion Laboratory, Pasadena, CA, August 29-30, 2002.

${ }^{4}$ Starr, B.R., Powell, R.W., Simulation, Monte Carlo, Performance, Neptune Aerocapture Systems Analysis Review, Marshall Space Flight Center, Huntsville, AL, October 28, 29, 2003.

${ }^{5}$ Dyke, R.E., "Planetary Probe Mass Estimation Tool Development and Its Application to Titan Aerocapture", paper AIAA-2003-4956 presented at the 39th AIAA/ASME/SAE/ASEE Joint Propulsion Conference and Exhibit, Huntsville, AL, July 22, 2003.

${ }^{6}$ Microsoft@ Excel 2002 SP3, Software Package, Microsoft Corporation, 2002.

${ }^{7}$ I-DEAS ${ }^{\circledR}$, PC Software Package, Ver. 9m2, Electronic Data Systems Corporation, Plano, TX, 2003.

${ }^{8}$ Collier Research HyperSizer ${ }^{\mathrm{TM}}$, Software Package, Ver. 4.0.7, Collier Research Corporation, Hampton, VA, 2002.

${ }^{9}$ HSLoad, Software Package, Ver. 1, NASA Langley Research Center, Hampton, VA, 2004.

${ }^{10}$ MSC/NASTRAN, Software Package, Ver 70, The MacNeal-Schwendler Corporation, Los Angeles, CA, 2000.
11 I-DEAS $®$ Fundamentals of Part Design Course Guide, EDS PLM Solutions, Milford, OH, 2001, p. 2.

${ }^{12}$ Collier Research Corporation, HyperSizer ${ }^{\mathrm{TM}}$ Object Modeler Programmer's Manual, 2003.

${ }^{13}$ Dyke, R.E., Mass Sensitivities, Titan Aerocapture Systems Analysis Review, Jet Propulsion Laboratory, Pasadena, CA, August 29-30, 2002.

${ }^{14}$ Laub, B., Thermal Protection (TPS), Titan Aerocapture Systems Analysis Review, Jet Propulsion Laboratory, Pasadena, CA, August 29-30, 2002.

${ }^{15}$ Hrinda, G.A., Aeroshell Structure, Titan Aerocapture Systems Analysis Review, Jet Propulsion Laboratory, Pasadena, CA, August 29-30, 2002.

${ }^{16}$ Noca, M.A., Mission Analysis, Titan Aerocapture Systems Analysis Review, Jet Propulsion Laboratory, Pasadena, CA, August 29-30, 2002.

${ }^{17}$ Dyke, R.E., The Titan Project: Conceptual Aeroshell Mass Estimation, The Titan Project Review, NASA Langley Research Center, July 10, 2003.

${ }^{18}$ Edquist, K.T., Configuration \& Aerodynamics, Neptune Aerocapture Systems Analysis Review, Marshall Space Flight Center, Huntsville, AL, October 28, 29, 2003.

${ }^{19}$ Dyke, R.E., Structures Concepts, Neptune Aerocapture Systems Analysis Review, Marshall Space Flight Center, Huntsville, AL, October 28, 29, 2003.

${ }^{20}$ Hrinda, G.A., Structure for the $5.5 \mathrm{~m}$ Ellipsled, Neptune Aerocapture Systems Analysis Review, Marshall Space Flight Center, Huntsville, AL, October 28, 29, 2003.

${ }^{21}$ Laub, B., Chen, Y.K., Thermal Protection (TPS), Neptune Aerocapture Systems Analysis Review, Marshall Space Flight Center, Huntsville, AL, October 28, 29, 2003.

${ }^{22}$ Dyke, R.E., Mass Sensitivities, Neptune Aerocapture Systems Analysis Review, Marshall Space Flight Center, Huntsville, AL, October 28, 29, 2003. 